\title{
Microgeographic Heterogeneity of Border Malaria During Elimination Phase, Yunnan Province, China, 2011-2013
}

\author{
Xin Xu, ${ }^{1}$ Guofa Zhou, ${ }^{1}$ Ying Wang, Yue Hu, Yonghua Ruan, Qi Fan, \\ Zhaoqing Yang, Guiyun Yan, Liwang Cui
}

\begin{abstract}
To identify township-level high-risk foci of malaria transmission in Yunnan Province, China, along the international border, we retrospectively reviewed data collected in hospitals and clinics of 58 townships in 4 counties during 2011-2013. We analyzed spatiotemporal distribution, especially hot spots of confirmed malaria, using geographic information systems and Getis-Ord $\mathrm{G}_{i}^{*}(\mathrm{~d})$ cluster analysis. Malaria incidence, transmission seasonality, and Plasmodium vivax: $P$. falciparum ratio remained almost unchanged from 2011 to 2013 , but heterogeneity in distribution increased. The number of townships with confirmed malaria decreased significantly during the 3 years; incidence became increasingly concentrated within a few townships. High-/low-incidence clusters of $P$. falciparum shifted in location and size every year, whereas the locations of high-incidence $P$. vivax townships remained unchanged. All high-incidence clusters were located along the China-Myanmar border. Because of increasing heterogeneity in malaria distribution, microgeographic analysis of malaria transmission hot spots provided useful information for designing targeted malaria intervention during the elimination phase.
\end{abstract}

$\mathrm{M}$ alaria, one of the most devastating infectious diseases, creates an enormous public health burden in the developing world (1). Since 2000, increased financial support has strengthened malaria control programs, leading to substantially reduced malaria incidence and death rates, even in the high-transmission areas of sub-Saharan Africa (1). The estimated worldwide malaria death rate declined by $45 \%$ during $2000-2012$. Of the 97 countries with malaria transmission in 2013, twelve are in the preelimination

Author affiliations: Kunming Medical University, Kunming, China (X. Xu, Y. Hu, Y. Ruan, Z. Yang); University of California, Irvine, California, USA (G. Zhou, G. Yan); Institute of Tropical Medicine, Third Military Medical University, Chongqing, China (Y. Wang); Dalian Institute of Biotechnology, Dalian, China (Q. Fan); Pennsylvania State University, University Park, Pennsylvania, USA (L. Cui)

DOI: http://dx.doi.org/10.3201/eid2208.150390 phase and 7, including China, are in the elimination phase. In early 2009, China's Ministry of Health presented its Revised National Malaria Strategy 2010-2015; this strategy was followed by the Malaria Elimination Action Plan for 2010-2020, in which the Ministry of Health laid out a strategy to eliminate malaria by $2020(2,3)$.

Control efforts, guided by the 1-3-7 strategy (reporting a malaria case within 1 day; confirming, treating, and investigating the case within 3 days; and delivering an appropriate public health response to prevent further transmission within 7 days) have drastically reduced malaria incidence in central China (4). As a result, malaria transmission is restricted to the southwestern Yunnan Province along the international borders (5-8). Currently, Plasmodium vivax is the predominant species of malaria parasites in China, and autochthonous $P$. falciparum occurs only in Yunnan Province $(7,8)$.

In 2012, Yunnan Province reported an annual malaria incidence of 7.4 cases $/ 100,000$ population (8). Yunnan Province borders 3 malaria-endemic countries: Myanmar, Laos, and Vietnam. Previous studies found that crossborder migration from Myanmar was the major source of importation/reintroduction of $P$. falciparum malaria in Yunnan Province (9). Therefore, the control strategy during the elimination phase must focus on eliminating local transmission and cross-border introduction.

Elimination strategies can differ profoundly from control strategies because they require prospective, accurate identification of transmission foci and rapid control responses (10-13). Earlier studies in China relied exclusively on retrospective data acquired from county-level hospital records $(5-8)$. The retrospective nature of these studies raises questions about diagnostic accuracy, whereas the county-level epidemiologic data provide limited spatial resolution. A county in China typically comprises many townships, sometimes $\approx 100$ villages and totaling $\approx 1$ million persons, and it might span $\approx 100 \mathrm{~km}$ (14-17), which limit the usefulness of county-level risk assessment for

${ }^{1}$ These authors contributed equally to this article. 
guiding targeted malaria control and local malaria elimination. Thus, for spatially heterogeneous malaria transmission, finer-scale mapping is essential for deploying elimination measures.

We aimed to use prospectively confirmed malaria data to identify high-risk foci of malaria transmission at the township level in Yunnan Province along the international border. Specifically, we wanted to locate the transmission hot spots and determine whether malaria transmission is heterogeneous at the township level. Our goal was to provide data to help guide targeted malaria control response during the malaria elimination phase.

\section{Methods}

\section{Study Area}

The study area comprised 58 townships in 4 counties (Tengchong, Yingjiang, Longchuan, and Ruili) along the China-Myanmar border in Yunnan Province (Figure 1). Each township has 1 government-run healthcare center consisting of an inpatient hospital and other administrative facilities and sometimes a few small village-level clinics. In accordance with government policy, malaria diagnosis and treatment are free, regardless of the patient's nationality or origin of residence. The study area spans $13,200 \mathrm{~km}^{2}$; the population was $\approx 1.3$ million in 2010 . Approximately $85 \%$ of residents live in rural areas, and most are farmers. The climate is subtropical; average maximum/minimum

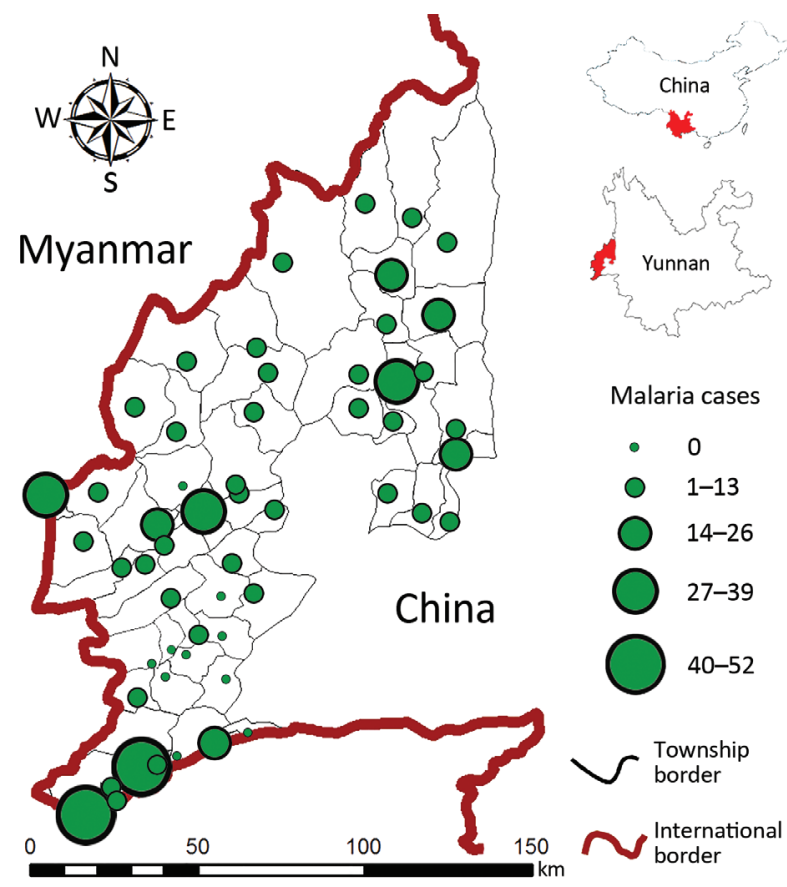

Figure 1. Locations of hospitals and healthcare centers (center of each circle) and total confirmed malaria cases in each township, Yunnan Province, China, 2011-2013. temperatures ranges from $13 / 4^{\circ} \mathrm{C}$ in January to $34 / 24^{\circ} \mathrm{C}$ in July. Annual rainfall in the study area is $\approx 2,200 \mathrm{~mm}$, with 1 rainy season during April July but no clear dry season. The topography is characterized by mountains separated by small, long, narrow basins dominated by rice fields. Among the 4 counties, Ruili is located in the largest basin of the study area. In 2012, Tengchong, Yingjiang, and Ruili counties were among China's leading 5 counties in malaria incidence (8).

\section{Data Collection}

We applied a prospective surveillance method and included all patients with fever who sought care at 58 local health centers and 4 hospitals in the study area during January 2011-December 2013. Local doctors screened persons with fever at local hospitals/clinics for malaria symptoms after patients signed consent or assent (for minors $<18$ years of age) forms (i.e., screening for suspected cases). A suspected malaria case was defined as malaria-related symptoms (fever with axillary temperature $\geq 37.5^{\circ} \mathrm{C}$, chills, severe malaise, headache, or vomiting) at examination or 1-2 days before examination. In brief, before sample collection, study procedures (i.e., sampling procedures, study benefits, and potential risks and discomforts) were explained to patients. Before samples were collected, demographic data such as sex, age, home address and name of participant, travel history during the preceding 14 days, malaria infections during the preceding 6 months and prescriptions obtained, and use of preventive measures were recorded. Identification numbers were used in the final analysis, and each patient's real identity and address was kept confidential. Blood samples were collected by fingerpricking. Labeled slides with thin and thick blood smears were prepared for microscopic Plasmodium species identification and parasite density counts. Thick and thin blood films were stained with $10 \%$ Giemsa and examined according to a previously described method (18). The hospitals where the patients were admitted confirmed all reported cases. The blood slides were reexamined by 3 experienced microscopists, who provided the final confirmation of malaria cases. For slides that were confirmed positive, the asexual parasite density and gametocyte density were scored against 200 leukocytes. Confirmed malaria was defined as malaria-related symptoms at examination or 1-2 days before examination and a Plasmodium-positive blood smear confirmed by microscopy. Persons in whom malaria was diagnosed were treated in accordance with national malaria treatment guidelines (http://www.who.int/malaria/ publications/atoz/9789241549127/en/).

\section{Data Analysis}

We calculated the malaria incidence rate as cases per 100,000 population per year or month based on 2010 
population census data. Nonlocal residents (e.g., visitors and travelers) were excluded from incidence rate calculation. We examined year-to-year differences in the number of townships where confirmed malaria was absent using a $\chi^{2}$ test. We tested township-level year-to-year differences in incidence using the Tukey-Kramer honest significant difference (HSD) of analysis of variance (ANOVA) post hoc test. P. vivax and P. falciparum case ratio for each year was also calculated.

We mapped spatial distribution of incidence rate at the township level using ArcGIS 10 (ESRI, Redlands, CA, USA). Spatial heterogeneity of confirmed malaria cases was measured with median incidence rate, range of incidence rate, Kurtosis, skewness, and coefficient of variation of incidence among townships. To determine whether the distance from the hospital or healthcare centers to the nearest border affected malaria incidence rate, we calculated distances from the hospital or healthcare centers to the nearest border using ArcGIS and divided them into 4 categories $(0-9,10-29,30-49$, and $\geq 50 \mathrm{~km})$; average incidence rate of $P$. falciparum and $P$. vivax were calculated for each distance group; and differences in incidence rate among different distance groups were compared using the Tukey-Kramer HSD of ANOVA post hoc test. To identify spatial clusters that might characterize the distribution of malaria at the China-Myanmar border, we used the GetisOrd local $G_{i}^{*}(d)$ test to determine the sizes and locations of high- and low-incidence clusters at the township level $(19,20)$. Getis-Ord $\mathrm{G}_{\mathrm{i}}{ }^{*}(\mathrm{~d})$ statistics have been commonly used for disease transmission hot spot analysis $(14,21-24)$. We examined hot spots separately for $P$. falciparum and $P$. vivax and annually so we could track temporal changes in transmission hot spot.

\section{Ethical Statement}

The institutional review boards of Kunming Medical University (Kunming, China); University of California, Irvine

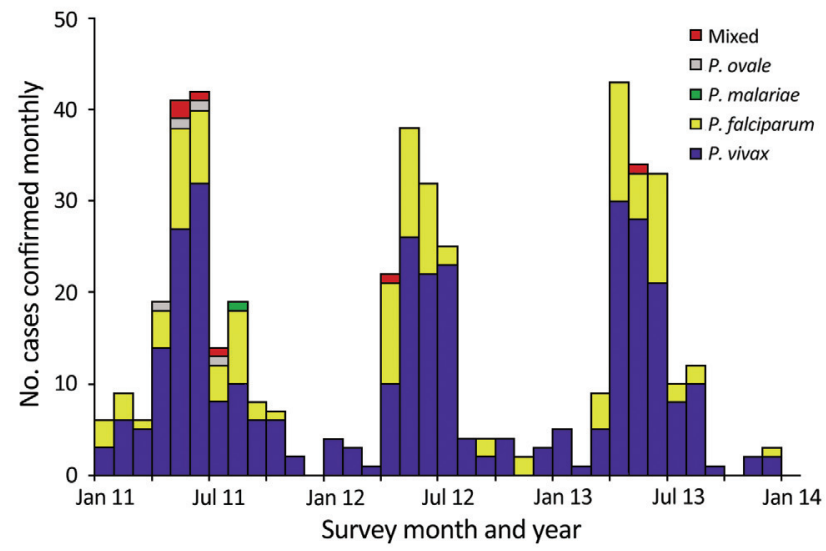

Figure 2. Monthly number of confirmed malaria cases of different Plasmodium species, Yunnan Province, China, 2011-2013.
(Irvine, CA, USA); and Pennsylvania State University (University Park, PA, USA) approved the study. We obtained written informed consent or assent (for minors $<18$ years of age) from all persons or parents or guardians who were willing to participate in the study.

\section{Results}

\section{Descriptive Statistics of Confirmed Malaria}

Blood smear examination by microscopy confirmed 468 malaria cases from the 915 suspected cases screened from $\approx 23,000$ febrile persons. The annual confirmed malaria incidence rate was 13.1 cases $/ 100,000$ population. We detected all 4 human Plasmodium species; $P$. vivax and $P$. falciparum accounted for 334 (71.4\%) and 123 (26.3\%), respectively. We found only $1(0.2 \%)$ P. malariae and 4 $(0.9 \%) P$. ovale infections. Six patients carried mixed infections by $P$. falciparum and $P$. vivax.

\section{Temporal Trend in Confirmed Malaria}

Confirmed malaria incidence showed strong seasonality and peaked during April-August each year (Figure 2). In December 2011 and October 2013, confirmed malaria infections were not detected in the study area, whereas during the peak months, incidence increased sharply to $\approx 40$ cases/month (3.3 cases/100,000 population/month) in each year of the study. P. vivax and $P$. falciparum parasites were detected nearly every month, but the other parasites and mixed infections were detected only during the high-transmission season (Figure 2). The P. vivax and $P$. falciparum case ratio remained almost unchanged: 2.5 for 2011, 2.6 for 2012, and 2.8 for 2013. The percentage of townships with no confirmed malaria increased from $43.1 \%$ in 2011 to $53.4 \%$ in 2012 and $67.2 \%$ in 2013 (Figure 3 ; Table), indicating a continuous reduction in malaria transmission in the area. This reduction also is reflected in the median incidence rates; the 0 median incidence rate

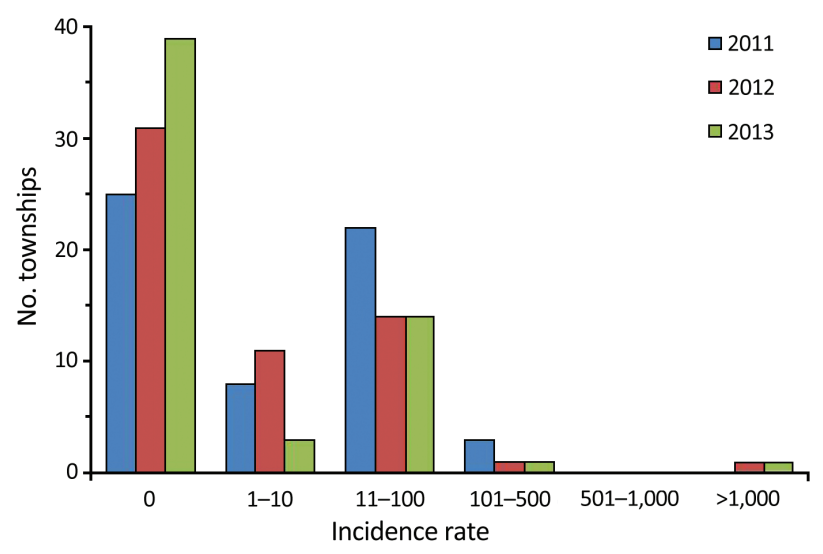

Figure 3. Malaria incidence (cases per 100,000 population), by year, Yunnan Province, China, 2011-2013. 
Table. Measurement of heterogeneous distribution of confirmed township-level malaria incidence rate, Yunnan Province, China, January 2011-December 2013

\begin{tabular}{|c|c|c|c|}
\hline \multirow[b]{2}{*}{ Parameter } & \multicolumn{3}{|c|}{ Year of surveillance } \\
\hline & 2011 & 2012 & 2013 \\
\hline No. townships with confirmed malaria* & 33 & 27 & 19 \\
\hline No. malaria cases & 170 & 144 & 149 \\
\hline \multicolumn{4}{|l|}{ Incidence rate† } \\
\hline Mean $(95 \% \mathrm{Cl})$ & $17.9(8.2-27.6)$ & $29.1(0-68.3)$ & $31.7(0-71.3)$ \\
\hline Median & 5.4 & 0 & 0 \\
\hline Range & 200 & 1133.3 & 1133.3 \\
\hline Kurtosis & 14.3 & 55.2 & 52.6 \\
\hline Skewness & 3.6 & 7.4 & 7.1 \\
\hline Coefficient of variation & 205.6 & 513.9 & 475.3 \\
\hline $\begin{array}{l}\text { *Significant differences }(p<0.05) \text { were deter } \\
\text { however, the difference between } 2011 \text { and } \\
\dagger \text { ?ases } / 100,000 \text { population/year. }\end{array}$ & en 2011 and 2012 & 2012 and 2 & not significant \\
\hline
\end{tabular}

in 2012 and 2013 indicated that $\approx 50 \%$ of townships had no confirmed malaria. The increased mean incidence rates from 2011 to 2013 reflected the increased heterogeneity of the distribution.

\section{Spatial Heterogeneity in Confirmed Malaria}

Malaria was increasingly concentrated in fewer townships, and the heterogeneous level increased among townships over time (Table). The annual township-level incidence rate of malaria ranged from 0 to 200.0 cases $/ 100,000$ population in 2011 and from 0 to $1,133.3$ cases/100,000 population in 2012 and 2013; however, the average incidence rate changed only from 17.9 to 31.7 cases/100,000 population annually from 2011 to 2013 (Table), and the increased mean incidence rate was caused primarily by a few extremely high-incidence townships. We detected malaria only from 33 townships during 2011, 27 during 2012, and 19 during 2013 (Figure 3). Coefficients of variation were well above $100 \%$ in all 3 years (Table). Spatial heterogeneity in incidence rate increased significantly over time (Table). These findings indicate a significantly heterogeneous distribution in township-level malaria incidence. Post hoc comparison indicated that differences in the annual average incidence rate at the township level were statistically insignificant among the 3 years (TukeyKramer HSD p $>0.05$ ), which indicated that the increasing mean annual incidences among townships from 2011 to 2013 most likely resulted from extreme variation in incidence rate among townships.

\section{Confirmed Malaria versus Distance to the Border}

The distance versus incidence rate analysis indicated that malaria incidence was 2- to 30-fold higher in areas within $10 \mathrm{~km}$ of the international border than it was in areas farther from the border, and the difference in incidence rate between the 2 categories increased during the 3 years. Within $10 \mathrm{~km}$ of the border, the annual $P$. vivax incidence was 21.48 during 2011, 57.89 during 2012, and 68.77 during 2013; for P. falciparum, incidence was 6.11during 2011,
13.04 during 2012, and 22.62 during 2013 (Figure 4). This finding indicated that confirmed malaria incidence was increasingly aggregated along the border. However, because of the huge variances in each group, statistical tests did not show any significant differences in the incidence rate among different distance groups (data not shown).

\section{Hot Spots of Confirmed Malaria}

The distribution of malaria incidence rates (Figure 5) and the clusters of low and high incidence rates (Figure 6) showing hot spots of confirmed malaria varied throughout
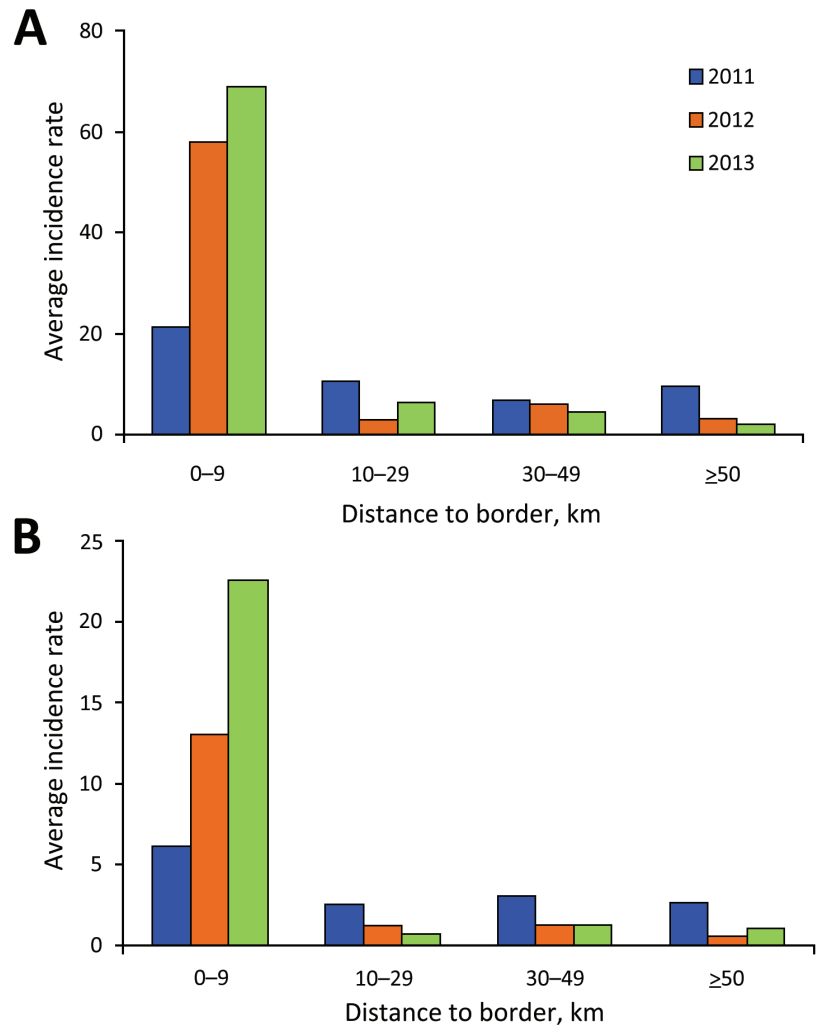

Figure 4. Malaria incidence (cases per 100,000 population) and distance to the nearest border, by year, Yunnan Province, China, 2011-2013. A) Plasmodium vivax. B) P. falciparum. 

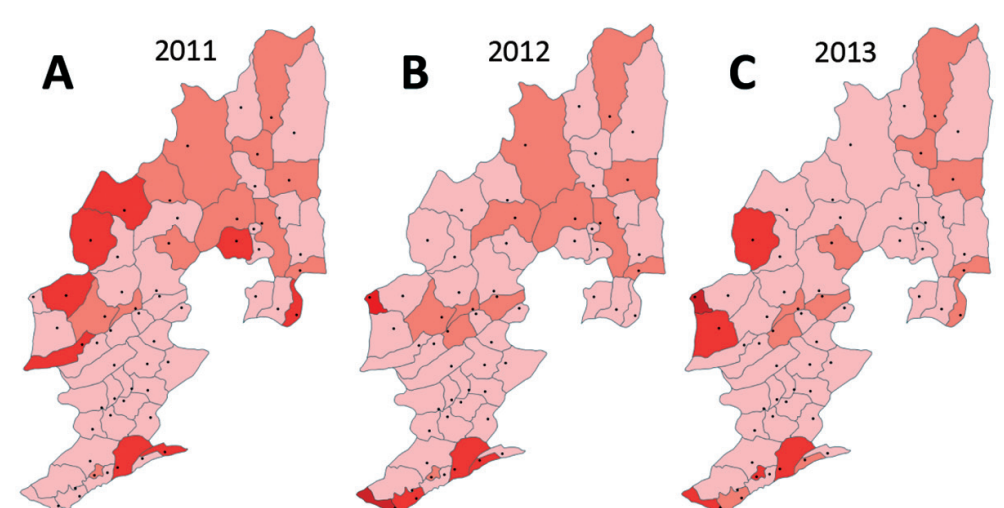

Incidence rate

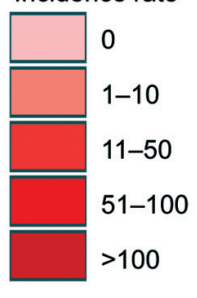

Figure 5. Distribution of townshiplevel malaria incidence rate (cases per 100,000 population), Yunnan Province, China, 2011-2013. A-C) Plasmodium falciparum. D-F) P. vivax.
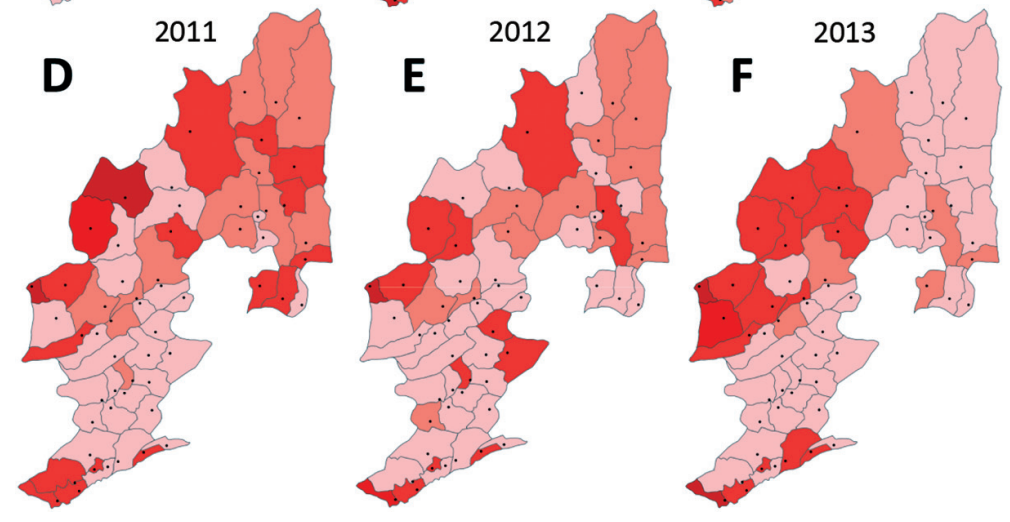

the years at the township level. Nevertheless, the transmission hot spots were generally located close to the international border.

The distribution maps of malaria incidence rates showed large spatial variation at the township level (Table; Figure 5). Townships with high incidences of $P$. vivax and $P$. falciparum were located mostly along the international border. However, many townships along the border had no confirmed malaria (Figure 5), illustrating the complex heterogeneous nature of malaria transmission in the border area. Compared with 2011, the geographic range of malaria incidence had shrunk in 2013. Clinical $P$. falciparum malaria cases were extremely scarce in 2013 (Figure 5, panel C), and $P$. vivax malaria cases were more aggregated in the central area along the border (Figure 5, panel F).

Results of the Getis-Ord $\mathrm{G}_{i}^{*}(\mathrm{~d})$ test indicated that all high-incidence clusters were located along the ChinaMyanmar border, namely Nabang, Xima, and Tongbiguan townships (Figure 6). The population of these 3 townships is $\approx 46,400$, and the area is $\approx 1,530 \mathrm{~km}^{2}$. However, for $P$. falciparum malaria, both the sizes and the exact locations of high- and low-incidence clusters shifted over time (Figure 6, panel A). On the other hand, high-incidence clusters of $P$. vivax remained in the same areas, although the size of clusters shrank from 2011 to 2012-2013. Coincidentally, high-incidence clusters comprised exactly the same townships for both $P$. falciparum and $P$. vivax in 2013 (Figure 6, panels $\mathrm{C}, \mathrm{F}$ ).

\section{Discussion}

China successfully reduced illness and death from malaria during the 1980s-2000s and is now in the malaria elimination phase. Strategies to achieve and maintain malaria elimination should concentrate on identifying and eliminating transmission foci through passive and active methods of case detection (12). As malaria transmission declines, the number and sizes of the infection foci are shrinking. Thus, finer-scale transmission maps are required to identify small transmission hot spots. Because the township is the smallest administrative unit in China that has government-run healthcare centers, transmission mapping at the township level might be the best approach for elimination planning.

In Yunnan Province, $P$. vivax, which is difficult to eliminate, has become the predominant parasite species. Therefore, as overall malaria incidence rates fell in a region, we would expect the proportion of $P$. vivax cases to increase (25). Previous studies found that clinical P. falciparum malaria in Yunnan Province accounted for $\approx 15 \%$ of total cases during 19912006 and 22\% during 20012005 $(26,27)$. However, we did not find further decrease in the proportion of P. falciparum malaria during 2011-2013, which accounted for $\approx 27 \%$ of total clinical cases. Most 

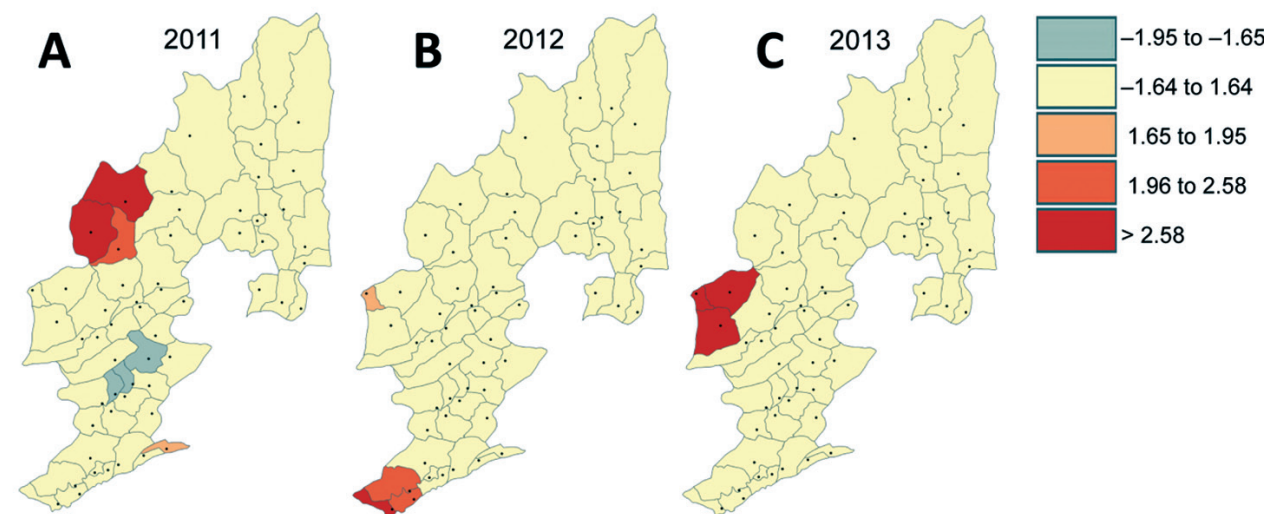

Figure 6. Clusters of low and high malaria incidence rates (cases per 100,000 population) detected at the township level and their shift over time, Yunnan Province, China, 2011-2013. A-C) Plasmodium falciparum. D-F) P. vivax.
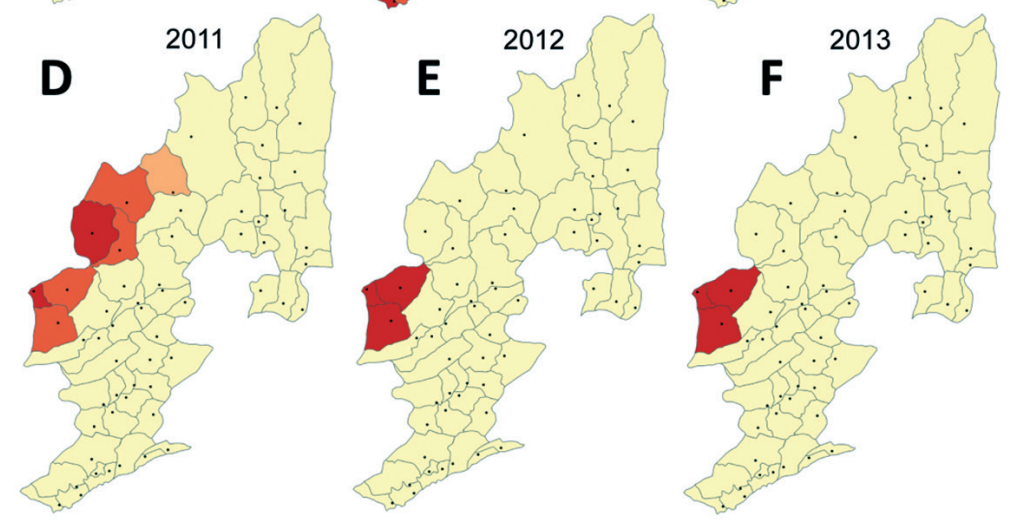

$P$. falciparum cases occurred along the international border. Importation probably explains this unusual increase in the $P$. falciparum proportion. A previous study conducted in the same area found that clinical P. falciparum malaria was significantly associated with cross-border travel, especially travel to Myanmar (9).

Because malaria transmission rates are much higher in Myanmar than in China $(9,28)$, persons living close to the border are at higher risk for infection. We found much a higher malaria incidence in the border area than in places farther from the border. Hot spot analysis found that high-incidence clusters of confirmed malaria were all located along the international border. Yet, the actual risk depends on the exact locations because malaria incidence rate in the border townships varied tremendously, from 0 to 1,133 cases/100,000 population annually in 2013 . In addition, the hot spots of malaria changed over time and differed between $P$. falciparum and $P$. vivax. The shift of $P$. falciparum transmission hot spots might again be strongly linked to the pattern of cross-border population migration $(9,28,29)$. The Getis-Ord local $G_{i}{ }^{*}(d)$ test has been commonly used to determine clustering of transmission hot spots of different diseases $(14,21-24)$; the test can be used in even finer-scale clustering analysis, such as village- or household-level hot spot analysis $(20,30,31)$. The use of spatial clustering combined with geographic information systems clearly shows the locations and the sizes of the transmission hot spots. If this method is implemented in real-time monitoring, the development of hot spots can be detected early, thus enabling targeted interventions in a timely manner. Thus, this method can become a conventional parameter in a programmatic decision-making process by disease prevention and control authorities. We emphasize that, for active case tracking at the elimination stage, such a hot spot analysis at the village or household level might be worth investigating, even though it demands more resources (31). In addition, the spatial shift in malaria incidence hot spots from year to year is a challenge for the national malaria control program. For the national malaria control program to identify hot spots of malaria incidence and target malaria control in a timely manner, the spatial cluster analysis will need to be performed more frequently.

Regardless of the changing epidemiology, malaria cases continued to exhibit a seasonal transmission pattern, peaking in April-July, mostly reflecting the dynamics of monthly rainfall in this region $(9,32-34)$. Seasonal migration patterns also might contribute to seasonal malaria transmission, as in other parts of the Greater Mekong Subregion such as western Thailand (35-38). Therefore, measures need to be developed to strengthen surveillance of cross-border migratory human populations to prevent malaria reintroduction. One limitation of our study is that we did not differentiate indigenous from definitive 
imported cases because of the lack of travel history information for many persons with confirmed malaria. The clear seasonality of malaria cases and migratory populations should be considered in future malaria prediction analyses so that targeted monitoring and control efforts can be deployed more precisely.

The complex heterogeneous distribution of low but focalized and mobilized confirmed malaria transmission brings enormous challenges to malaria elimination plans. Strategies for malaria elimination in China should focus on these few transmission foci. For example, vector control measures should be enhanced in these areas; active case surveillance should be deployed to track the transmission; and more rigorous case management strategies should be implemented. In addition, Myanmar is considered the major source of parasite importation in the entire Greater Mekong Subregion $(39,40)$, and human migration in the area plays an important role in malaria transmission in China (35-38; http://www.searo.who.int/entity/malaria/ documents/Mekong_pro/en/). Therefore, future elimination efforts should focus on the effects of cross-border activities on malaria parasite transmission, and strategies should include more intensive surveillance so that prevention and control activities can be directed at hot spot regions along the China-Myanmar border.

\section{Acknowledgments}

We thank the field team members for their technical assistance. We are grateful to the communities and hospitals for their willingness to participate in this research.

This project was funded by the National Institutes of Health (U19 AI089672 to L.C., G.Z., and G.Y.); the National Science Foundation of China (nos. U1202226 and 31260508 to Z.Y.); and the Ministry of Education of China (no. 20125317110001 to Z.Y.).

$\mathrm{Ms} . \mathrm{Xu}$ is a graduate student in Kunming Medical University, Kunming, China. Her research interests are drug resistance and epidemiology of malaria.

\section{References}

1. World Health Organization. World malaria report 2014 [cited 2015 Feb 15]. http://www.who.int/malaria/publications/world_malaria_ report 2014/en/

2. Ministry of Health of the People's Republic of China. From malaria control to eliminate: a revised national malaria strategy 2010-2015. Beijing: Ministry of Health of the People's Republic of China; 2009.

3. Ministry of Health of the People's Republic of China. Action plan of China malaria elimination (2010-2020) [cited 2015 Feb 15]. http://www.malariaeradication.org/elimination-programmes/ peoples-republic-china

4. Cao J, Sturrock HJ, Cotter C, Zhou S, Zhou H, Liu Y, et al. Communicating and monitoring surveillance and response activities for malaria elimination: China's "1-3-7" strategy. PLoS Med. 2014;11:e1001642. http://dx.doi.org/10.1371/journal. pmed. 1001642
5. Clements AC, Barnett AG, Cheng ZW, Snow RW, Zhou HN. Space-time variation of malaria incidence in Yunnan Province, China. Malar J. 2009;8:180. http://dx.doi.org/10.1186/1475-2875$8-180$

6. Lin H, Lu L, Tian L, Zhou S, Wu H, Bi Y, et al. Spatial and temporal distribution of falciparum malaria in China. Malar J. 2009;8:130. http://dx.doi.org/10.1186/1475-2875-8-130

7. Zhou SS, Wang Y, Li Y. Malaria situation in the People's Republic of China in 2010 [in Chinese]. Zhongguo Ji Sheng Chong Xue Yu Ji Sheng Chong Bing Za Zhi. 2011;29:401-3.

8. Xia ZG, Feng J, Zhou SS. Malaria situation in the People's Republic of China in 2012 [in Chinese]. Zhongguo Ji Sheng Chong Xue Yu Ji Sheng Chong Bing Za Zhi. 2013;31:413-8.

9. Zhou G, Sun L, Xia R, Duan Y, Xu J, Yang H, et al. Clinical malaria along the China-Myanmar border, Yunnan Province, China, January 2011-August 2012. Emerg Infect Dis. 2014;20:675-8. http://dx.doi.org/10.3201/eid2004.130647

10. Bejon P, Williams TN, Liljander A, Noor AM, Wambua J, Ogada E, et al. Stable and unstable malaria hotspots in longitudinal cohort studies in Kenya. PLoS Med. 2010;7:e1000304. http://dx.doi.org/10.1371/journal. pmed.1000304

11. Bousema T, Drakeley C, Gesase S, Hashim R, Magesa S, Mosha F, et al. Identification of hot spots of malaria transmission for targeted malaria control. J Infect Dis. 2010;201:1764-74. http://dx.doi.org/10.1086/652456

12. Moonen B, Cohen JM, Snow RW, Slutsker L, Drakeley C, Smith DL, et al. Operational strategies to achieve and maintain malaria elimination. Lancet. 2010;376:1592-603. http://dx.doi.org/10.1016/S0140-6736(10)61269-X

13. Bousema T, Griffin JT, Sauerwein RW, Smith DL, Churcher TS, Takken W, et al. Hitting hotspots: spatial targeting of malaria for control and elimination. PLoS Med. 2012;9:e1001165. http://dx.doi.org/10.1371/journal.pmed.1001165

14. Dhimal M, O'Hara RB, Karki R, Thakur GD, Kuch U, Ahrens B. Spatio-temporal distribution of malaria and its association with climatic factors and vector-control interventions in two high-risk districts of Nepal. Malar J. 2014;13:457. http://dx.doi.org/10.1186/1475-2875-13-457

15. Yin JH, Zhou SS, Xia ZG, Wang RB, Qian YJ, Yang WZ, et al. Historical patterns of malaria transmission in China. Adv Parasitol. 2014;86:1-19. http://dx.doi.org/10.1016/B978-0-12-800869$0.00001-9$

16. Zhou XN, Xia ZG, Wang RB, Qian YJ, Zhou SS, Utzinger J, et al. Feasibility and roadmap analysis for malaria elimination in China. Adv Parasitol. 2014;86:21-46. http://dx.doi.org/10.1016/B978-012-800869-0.00002-0

17. Yin JH, Yan H, Huang F, Li M, Xiao HH, Zhou SS, et al. Establishing a China malaria diagnosis reference laboratory network for malaria elimination. Malar J. 2015;14:40. http://dx.doi.org/10.1186/s12936-015-0556-z

18. Tebit KE. Comparison of capillary and venous blood using blood film microscopy in the detection of malaria parasites: a hospital based study. Scientific J Microbiology. 2013;2:89-94.

19. Zhou G, Sirichaisinthop J, Sattabongkot J, Jones J, Bjørnstad ON, Yan G, et al. Spatio-temporal distribution of Plasmodium falciparum and $P$. vivax malaria in Thailand. Am J Trop Med Hyg. 2005;72:256-62.

20. Zhou G, Munga S, Minakawa N, Githeko AK, Yan G. Spatial relationship between adult malaria vector abundance and environmental factors in western Kenya highlands. Am J Trop Med Hyg. 2007;77:29-35.

21. Kelly-Hope LA, Hemingway J, McKenzie FE. Environmental factors associated with the malaria vectors Anopheles gambiae and Anopheles funestus in Kenya. Malar J. 2009;8:268. http://dx.doi.org/10.1186/1475-2875-8-268 
22. Cartabia M, Campi R, Clavenna A, Bortolotti A, Fortino I, Merlino L, et al. Geographical epidemiology of antibacterials in the preschool age. Int J Health Geogr. 2012;11:52. http://dx.doi.org/10.1186/1476-072X-11-52

23. Haque U, Scott LM, Hashizume M, Fisher E, Haque R, Yamamoto T, et al. Modelling malaria treatment practices in Bangladesh using spatial statistics. Malar J. 2012;11:63. http://dx.doi.org/10.1186/1475-2875-11-63

24. Ruktanonchai CW, Pindolia DK, Striley CW, Odedina FT, Cottler LB. Utilizing spatial statistics to identify cancer hot spots: a surveillance strategy to inform community-engaged outreach efforts. Int J Health Geogr. 2014;13:39. http://dx.doi.org/10.1186/1476-072X-13-39

25. Vogel G. The forgotten malaria. Science. 2013;342:684-7. http://dx.doi.org/10.1126/science.342.6159.684

26. Li HX, Chen GW, Yang YC, Jiang H. Malaria situation in Yunnan Province during 2001-2005 [in Chinese]. Zhongguo Ji Sheng Chong Xue Yu Ji Sheng Chong Bing Za Zhi. 2008; 26:46-9.

27. Hui F-M, Xu B, Chen Z-W, Cheng X, Liang L, Huang H-B, et al. Spatio-temporal distribution of malaria in Yunnan Province, China. Am J Trop Med Hyg. 2009;81:503-9.

28. Li N, Parker DM, Yang Z, Fan Q, Zhou G, Ai G, et al. Risk factors associated with slide positivity among febrile patients in a conflict zone of north-eastern Myanmar along the China-Myanmar border. Malar J. 2013;12:361. http://dx.doi.org/10.1186/1475-2875-12-361

29. Kumar A, Chery L, Biswas C, Dubhashi N, Dutta P, Dua VK, et al. Malaria in South Asia: prevalence and control. Acta Trop. 2012;121:246-55. http://dx.doi.org/10.1016/j.actatropica.2012.01.004

30. Getis A, Morrison AC, Gray K, Scott TW. Characteristics of the spatial pattern of the dengue vector, Aedes aegypti, in Iquitos, Peru. Am J Trop Med Hyg. 2003;69:494-505.

31. Hu Y, Zhou G, Ruan Y, kLee MC, Xu X, Deng S, et al. Seasonal dynamics and microgeographical spatial heterogeneity of malaria along the China-Myanmar border. Acta Trop. 2016;157:12-9. http://dx.doi.org/10.1016/j.actatropica.2016.01.022

32. Bi Y, Hu W, Yang H, Zhou X-N, Yu W, Guo Y, et al. Spatial patterns of malaria reported deaths in Yunnan Province, China. Am J Trop Med Hyg. 2013;88:526-35. http://dx.doi.org/10.4269/ ajtmh.2012.12-0217
33. Lu G, Zhou S, Horstick O, Wang X, Liu Y, Müller O. Malaria outbreaks in China (1990-2013): a systematic review. Malar J. 2014;13:269. http://dx.doi.org/10.1186/1475-2875-13-269

34. Zhao X, Chen F, Feng Z, Li X, Zhou X-H. Characterizing the effect of temperature fluctuation on the incidence of malaria: an epidemiological study in south-west China using the varying coefficient distributed lag non-linear model. Malar J. 2014;13:192. http://dx.doi.org/10.1186/1475-2875-13-192

35. Wiwanitkit V. High prevalence of malaria in Myanmar migrant workers in a rural district near the ThailandMyanmar border. Scand J Infect Dis. 2002;34:236-7. http://dx.doi.org/10.1080/00365540110077272

36. Richards AK, Banek K, Mullany LC, Lee CI, Smith L, Oo EKS, et al. Cross-border malaria control for internally displaced persons: observational results from a pilot programme in eastern Burma/Myanmar. Trop Med Int Health. 2009;14:512-21. http://dx.doi.org/10.1111/j.1365-3156.2009.02258.x

37. Coker RJ, Hunter BM, Rudge JW, Liverani M, Hanvoravongchai P. Emerging infectious diseases in southeast Asia: regional challenges to control. Lancet. 2011;377:599-609. http://dx.doi.org/10.1016/ S0140-6736(10)62004-1

38. Jitthai N. Migration and malaria. Southeast Asian J Trop Med Public Health. 2013;44(Suppl 1):166-200, discussion 306-7.

39. Cui L, Yan G, Sattabongkot J, Cao Y, Chen B, Chen X, et al. Malaria in the Greater Mekong Subregion: heterogeneity and complexity. Acta Trop. 2012;121:227-39.

http://dx.doi.org/10.1016/j.actatropica.2011.02.016

40. Cui L, Yan G, Sattabongkot J, Chen B, Cao Y, Fan Q, et al. Challenges and prospects for malaria elimination in the Greater Mekong Subregion. Acta Trop. 2012;121:240-5. http://dx.doi.org/10.1016/j.actatropica.2011.04.006

Address for correspondence: Zhaoqing Yang, Department of Pathogen Biology and Immunology, Kunming Medical University, Kunming, China; email: zhaoqingy92@hotmail.com; Guiyun Yan, Program in Public Health, University of California, Irvine, Irvine, CA 92697, USA; email: guiyuny@uci.edu; Liwang Cui, Department of Entomology, Pennsylvania State University, University Park, PA 16802, USA; email: luc2@psu.edu

\section{Get the content you want delivered to your inbox.}
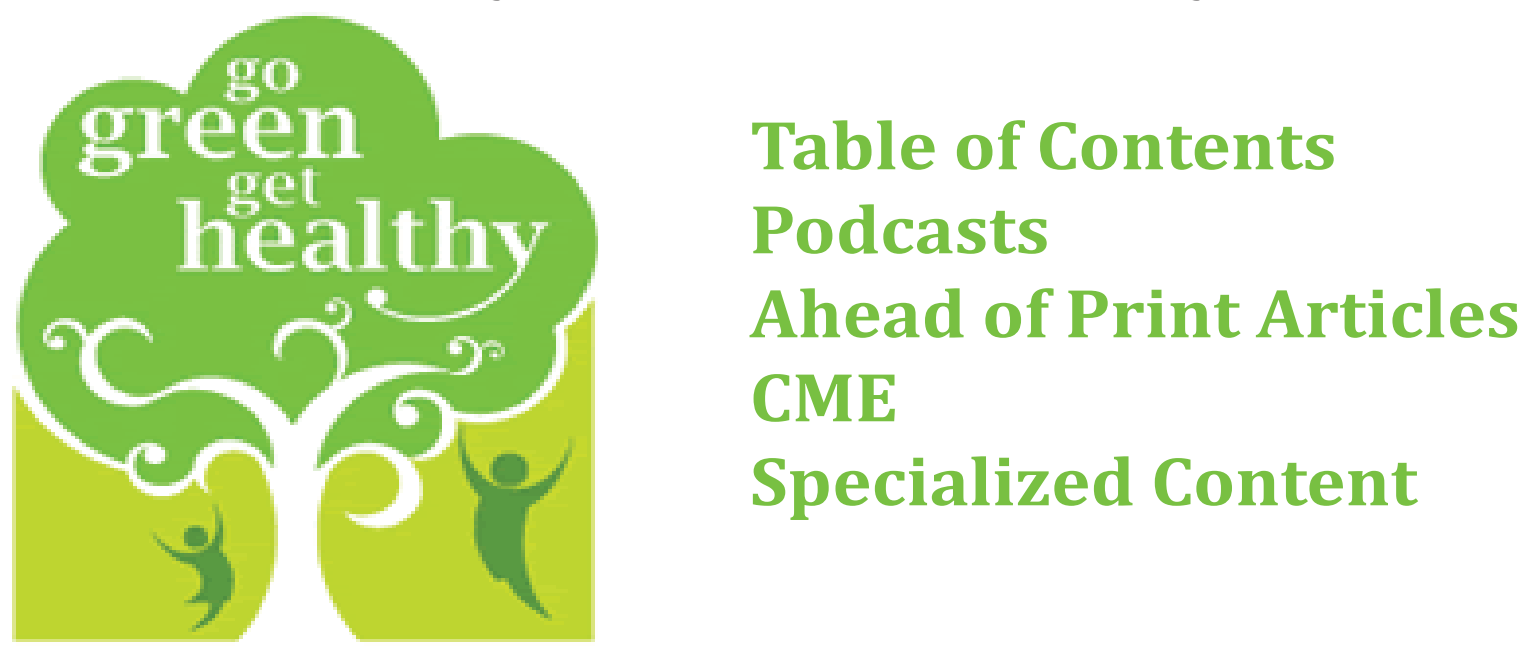

Online subscription: wwwnc.cdc.gov/eid/subscribe.htm 